\title{
Aportes en investigación formativa: caso de estudio de los semilleros de investigación en el programa de Ingeniería en Informática
}

\author{
Contributions in formative research: case study of research \\ incubators in the Computer Engineering program
}

Citar como: Silva Monsalve, A. M., Roa Banquez, K., Vásquez Morales, G., Saya Acevedo, E. H., Sierra Cantor, L. F., Bohórquez Ramírez, G. y López Moyano, E. (2020). Aportes en investigación formativa: caso de estudio de los semilleros de investigación en el programa de Ingeniería en Informática. CITAS, 6(1). https://doi.org/10.15332/24224529.6363

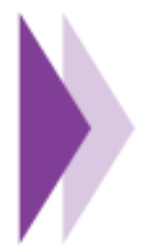

Alexandra María Silva Monsalve ${ }^{1}$; Katherine Roa Banquez ${ }^{2}$; Geraldyn Vásquez Morales ${ }^{3}$; Elder Hernán Saya Acevedo $^{4}$; Luis Fernando Sierra Cantor ${ }^{5}$; Gabriela Bohórquez Ramírez ${ }^{6}$; Edwin López Moyano ${ }^{7}$

1 Facultad de Ciencias y Tecnologías de la Universidad Santo Tomás 2 Facultad de Ciencias y Tecnologías de la Universidad Santo Tomás 3 Facultad de Ciencias y Tecnologías de la Universidad Santo Tomás 4 Facultad de Ciencias yTecnologías de la Universidad Santo Tomás

5 Facultad de Ciencias yTecnologías de la Universidad Santo Tomás

6 Facultad de Ciencias yTecnologías de la Universidad Santo Tomás

7 Facultad de Ciencias yTecnologías de la Universidad Santo Tomás

Correo electrónico: alexandrasilva@ustadistancia.edu.co katherinroa@ustadistancia.edu.co yasbleydyvasquez@ustadistancia.edu.co eldersaya@ustadistancia.edu.co luissierrac@ustadistancia.edu.co gabrielabohorquez@ustadistancia.edu.co edwin.lopez@ustadistancia.edu.co 


\section{Resumen}

El objetivo de la reflexión fue indagar en las acciones que, por medio de la investigación formativa, se pueden adelantar en los semilleros de investigación. Inicialmente, se destaca la importancia que la investigación ha tenido en la transformación de la sociedad. De esta manera, se precisa en los aportes de la investigación formativa y la formación en investigación como retos de las instituciones de educación superior. Metodológicamente se adelantó una investigación documental de tipo exploratorio, que se desarrolló en varias fases. En la primera se realizó una exploración en la base de datos Scopus, en la que se indagó por trabajos en investigación formativa en diferentes áreas. Seguidamente se identificaron los proyectos adelantados por los estudiantes en su participación en el semillero, y se revisó cómo sus resultados han aportado a los objetivos de los semilleros, pero principalmente a la investigación formativa en el programa de Ingeniería en Informática. Se puede concluir que las contribuciones de los semilleros han aportado a diversas áreas del conocimiento.

Palabras clave: investigación, formación en investigación, semilleros, sociedad, transformación.

El desarrollo expositivo de este artículo se orienta en dos fases. La primera es resaltar la importancia que ha tenido la investigación como herramienta para la transformación de la sociedad. La segunda es el papel de las instituciones educativas, que deben propender por el desarrollo y fortalecimiento de las habilidades investigativas. Se inicia la reflexión con lo mencionado por Hernández, Fernández, y Baptista (2014): "Dios ha concedido a la humanidad la capacidad de investigar, está en nosotros lograr que sea una herramienta para crear un mundo mejor y facilitar el bienestar integral de todos los seres humanos" (p. 18).

\section{Transformación de la sociedad e investigación}

La Revolución Industrial transformó la economía mundial. Parte de estos cambios se deben a la influencia de la ciencia y la técnica con la que fueron abordados los procesos productivos. A propósito de lo referido por algunos autores, que cada época trae sus propias máquinas, la humanidad ha experimentado cambios constantes que inician con la revolución cognitiva, en la cual el hombre, debido de la necesidad de establecer contacto, desarrolló el lenguaje; seguida de la revolución agrícola, que trajo consigo el cambio de una población de nómadas a sedentarios; luego, la Revolución Industrial permitió la transformación en diferentes sectores y procesos de la economía.

Asimismo, la Revolución Industrial ha tenido diferentes etapas. Entre 1850 y 1913 se dio la Segunda Revolución Industrial con el advenimiento del acero, el petróleo y la electricidad. La Tercera Revolución, o revolución digital (1980-2012), incorporó las tecnologías de la información y la comunicación (TIC). Luego, la Cuarta Revolución Industrial se caracteriza por la integración de las transformaciones digitales en las sociedades (Flórez, Aguilera y Salcedo, 2019, p. 1), la cual va de la mano con la Industria 4.0, que incorpora el aprendizaje automatizado, la inteligencia artificial, la nanotecnología, la computación cuántica, la biotecnología, la impresión 3D, el internet de las cosas (IoT, por sus siglas en inglés) (Ministerio de Ciencia [Minciencias], 2019).

Lo anterior deja ver cómo la investigación se convierte en una herramienta que conduce al avance y a la transformación de la sociedad. Por medio de esta es posible buscar respuestas a los interrogantes que surgen de las necesidades constantes y

\section{Abstract}

The objective of the reflection was to investigate the actions that, through formative research, can be carried out in research incubators. Initially, the importance that research has had in the transformation of society is highlighted. In this way, the contributions of formative research and research training are specified as challenges for higher education institutions. Methodologically, an exploratory documentary research was carried out, developed in several phases. In the first phase, an exploration of the Scopus database was made, inquiring about formative research works in different areas. Next, the projects carried out by the students in their participation in the incubators were identified, and it was reviewed how their results have contributed to the objectives of the incubators, but mainly to the formative research in the Computer Engineering program. It can be concluded that incubators have contributed to various areas of knowledge.

Keywords: research, research training, incubators, society, transformation.

cambiantes en la modernidad. Así, desde épocas ancestrales el ser humano se inquietó sobre la búsqueda de soluciones a sus necesidades. Con el pasar de los años, los diferentes adelantos tecnológicos permitieron que estas se suplieran por medio de la incorporación de la ciencia y la técnica.

\section{Acciones universitarias para el fomento de la investigación}

El compromiso de las universidades debe orientarse a contribuir en la formación de capital humano que incentive en sus estudiantes habilidades investigativas para la consolidación de un pensamiento científico. En Colombia, por parte del Ministerio de Ciencias, a través del ecosistema científico, se definieron acciones para mejorar la calidad de las instituciones de educación superior. Se implementaron alianzas que contribuyen al desarrollo regional para enfrenar los desafíos sociales y productivos del país mediante el aporte de recursos para la investigación (Minciencias, 2020).

Asimismo, las instituciones universitarias proponen diversas estrategias para incentivar y desarrollar la investigación, algunas se orientan en recursos para proyectos, formación en capacidades en Ciencia, Tecnología e Innovación (CTeI). En particular, la Universidad Santo Tomás busca fortalecer estas capacidades mediante la generación y desarrollo de estrategias pedagógicas, también con la intención de vincular estudiantes egresados en este tipo de estrategias (Universidad Santo Tomás, 2020). Igualmente, se debe propender por el fomento de una cultura investigativa; esta se puede incentivar por medio de la formación investigativa y la investigación formativa. La primera se orienta a formar en investigación y para la investigación "en actividades investigativas que incorporan la lógica de la investigación y aplican métodos de investigación, pero que no implican necesariamente el desarrollo de proyectos de investigación completos ni el hallazgo de conocimiento nuevo y universal" (Restrepo, 2004, p. 3). La segunda, se ubica en formar para la investigación; esto, mediante actividades y acciones orientadas a cursos de investigación, lectura y discusión de informes de investigación.

En concordia con las estrategias que se proponen en la investigación formativa, los semilleros de investigación constituyen una oportunidad para que los estudiantes desarrollen 
sus competencias científicas. En este sentido, Villalba y González (2017) los definen como un "espacio que permite a sus integrantes, estudiantes y docentes una participación real, controlada, guiada y procesual del binomio enseñanza-aprendizaje que prioriza la libertad, la creatividad y la innovación para el desarrollo de nuevos esquemas mentales y métodos de aprendizaje" (p. 1). Por lo tanto, se consideran escenarios donde el estudiante, además de incentivar sus competencias investigativas, contribuye al libre progreso de la creatividad y la innovación (Castiblanco y López, 2020).

Sin embargo, incentivar la investigación en los estudiantes es un reto para las instituciones educativas. Lo anterior, por diversas situaciones, entre estas, el escaso conocimiento en investigación por parte de los estudiantes. Por otra parte, la dificultad en la interacción que se debe generar entre los participantes para construir procesos colaborativos. En este orden de ideas, los programas académicos en modalidades a distancia o en línea carecen de espacios de encuentros presenciales. Aunque existen puntos de encuentro, son limitados porque los estudiantes en este tipo de modalidades se caracterizan por trabajar de manera individual, al tener en cuenta que sus ocupaciones personales y laborales limitan su participación en grupos de trabajo.

En particular, el programa de Ingeniería en Informática de la Facultad de Ciencias y Tecnologías de la Universidad Santo Tomás (Bogotá, Colombia) ha incentivado la investigación formativa mediante los semilleros de investigación. Desde la creación del programa se ha contado con diferentes semilleros, entre estos, el Semillero Innovapp, el cual tenía como objetivo generar propuestas en el diseño y desarrollo de software, así como el desarrollo de recursos didácticos multimedial para la inclusión de personas con discapacidad; el Semillero Siderapp se fundamentaba en promover la cooperación investigativa de los estudiantes en las diferentes propuestas de investigación implementando desarrollos de software y aplicaciones móviles que mejoren la tecnología en los diferentes contextos. Estos fueron trabajados durante el periodo del 2014 al 2019.

En la actualidad se cuenta con el Semillero Green TIC, el cual está activo desde el año 2013 y pretende impulsar propuestas de investigación y desarrollo que posibiliten proporcionar una solución a problemas concretos atañidos con los sistemas informáticos y telemáticos, haciendo énfasis en la preservación del medio ambiente. Estos permiten la actualización constante de las prácticas educativas y el currículo, así como la formación integral de los estudiantes y su proyección en el mundo globalizado. En el 2018 se da inicio con el Semillero Tecnologías Emergentes aplicadas a la Gestión de la Información y del Conocimiento TEAGIC, cuyo objetivo es presentar propuestas de innovación en la gestión de la información y del conocimiento mediada por tecnologías de la información y la comunicación (TIC). Estas estrategias pedagógicas en CTeI tienen como propósito orientar a los estudiantes en los procesos investigativos, proponer y aplicar propuestas en temas de investigación desde el avance de la tecnología y su integración en contextos educativos. Lo anterior con el objetivo de consolidar una propuesta mediada por los avances tecnológicos y la educación para la generación de una formación integral y fortalecida en las regiones donde el programa de Ingeniería en Informática tiene su área de actuación.

\section{Metodología}

Metodológicamente se adelantó una investigación documental de tipo exploratorio, con un enfoque cualitativo. La investigación se orientó en cuatro fases: en la primera se realizó una revisión documental en temas de investigación formativa, en la segunda etapa se presentan algunos de los trabajos de los estudiantes adelantados en los semilleros, y en la tercera se hace un análisis de la pertinencia de los trabajos que se adelantan en los semilleros del programa de Ingeniería en Informática.

De esta manera, en la revisión documental se indagó en la base de datos Scopus; se obtuvieron 29 resultados en información relacionada con trabajos de investigación formativa. En la figura 1 se muestran los resultados de trabajos de investigación formativa en diferentes áreas, en las cuales se evidencia que en su gran mayoría corresponden al área de las ciencias sociales (55.3\%); y los demás se ubican en las áreas de medicina, administración, ciencia de datos, ingeniería, estadística, artes y humanidades, y enfermería. 
Figura 1. Resultados de trabajos en investigación formativa por áreas

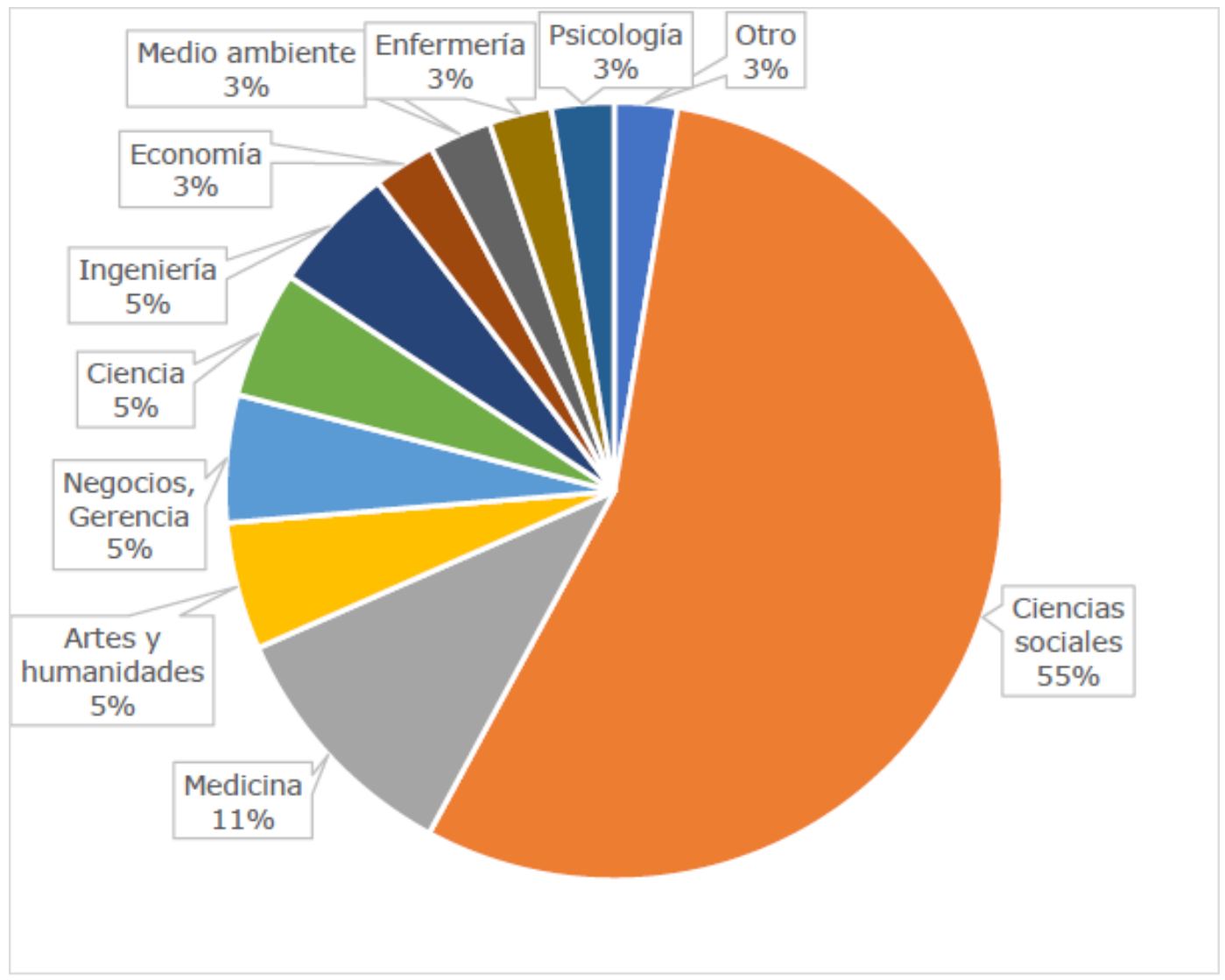

Fuente: elaboración propia con datos de Scopus (2020).

Los resultados anteriores evidencian un camino que apenas está iniciando con respecto a la implementación de la investigación formativa. Aunque en algunas áreas se tienen mayores avances, todavía se debe seguir trabajando e incentivando la investigación en los estudiantes. También, es un aspecto positivo el aumento de trabajos en investigación formativa, evolucionado con el paso de los años (figura 2). 
Figura 2. Evolución de trabajos en investigación formativa

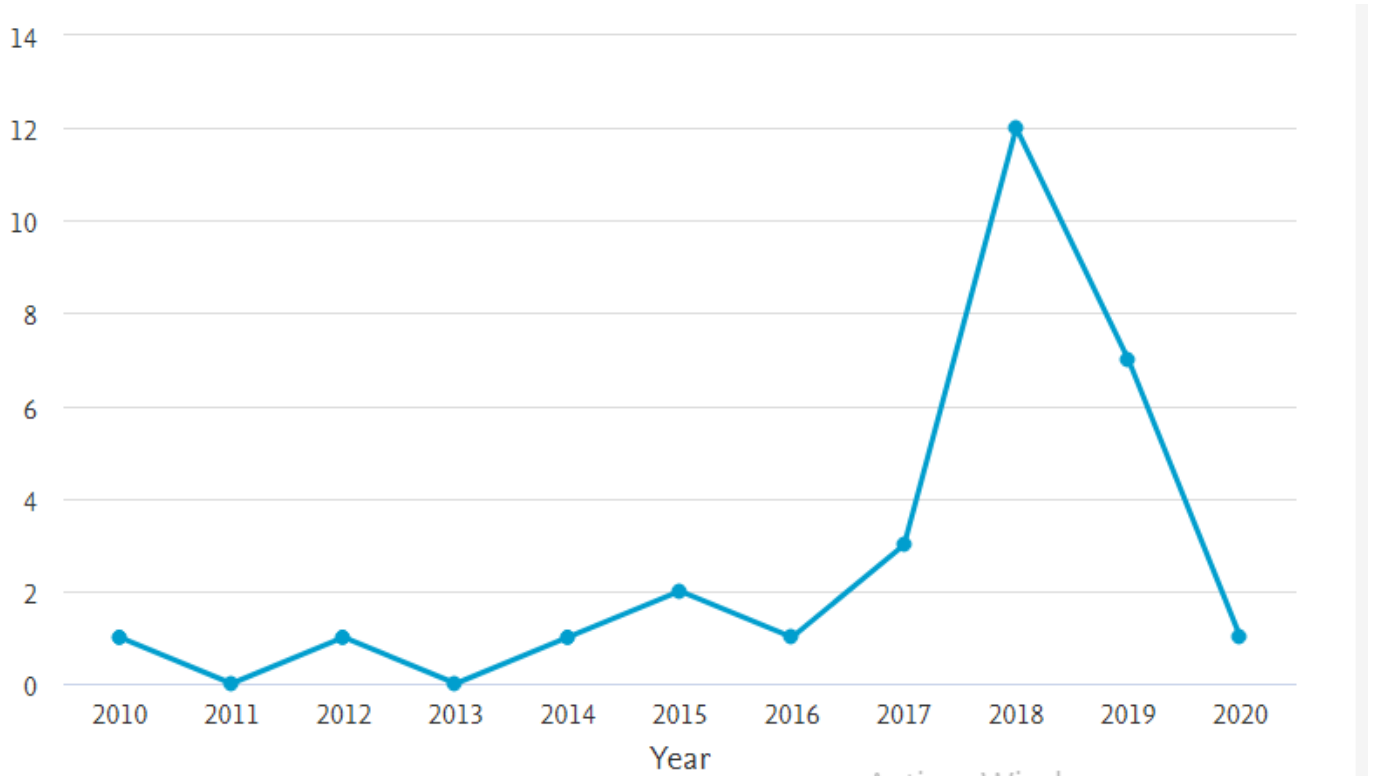

Fuente: elaboración propia con datos de Scopus (2020).

En la segunda fase se indaga por los trabajaos que han adelantado los estudiantes, se interroga en cómo los proyectos han dado respuesta a diversas áreas del conocimiento, entendiendo que la informática aporta herramientas que pueden contribuir transversalmente. De aquí en adelante se describen los resultados de los trabajos adelantados en cada uno de los semilleros. Por una parte, se tuvieron en cuenta los trabajos más destacados y también los estudiantes que por iniciativa se postularon a presentar los resultados de sus trabajos.

En el semillero TEAGIC se busca fortalecer a los estudiantes, tanto en investigación formativa como en formación investigativa. Se han vinculado con proyectos que se derivan de investigaciones propuestas por los docentes, así como la capacitación en procesos de investigación y disciplinares; estos últimos provienen desde iniciativas de los mismos participantes.

\section{Implementación de un software neurodidáctico}

El objetivo del proyecto fue la implementación de un software como herramienta para el fortalecimiento de los procesos lógicomatemáticos en los estudiantes de Ingeniería. El problema se identificó en la ausencia de habilidades en el pensamiento lógicomatemático, también se buscó la incursión de las TIC en los procesos de enseñanza-aprendizaje. Se establecieron diversas estrategias en cuanto a su integración curricular y a los beneficios que podrían tener en el desempeño de los estudiantes.

De esta manera, la incorporación de nuevas tecnologías en las aulas requiere de un esfuerzo sistemático por parte del docente para crear un ambiente propicio que incentive al estudiante para que pueda construir su conocimiento. Esto se convierte en un instrumento que favorece el desarrollo de nuevas prácticas. No necesariamente significa inventar algo nuevo, más bien se refiere a una nueva manera de hacer las cosas utilizando este conjunto de técnicas como una plataforma para el aprendizaje. La incorporación de las TIC en los procesos de fortalecimiento del pensamiento lógico-matemático constituye uno de los temas más importantes en la educación en ingeniería. Por tal razón, es importante que las universidades ofrezcan currículos flexibles, en donde las metodologías y herramientas de enseñanza estén orientadas a crear situaciones de aprendizaje que potencialicen el dominio del componente matemático como una herramienta imprescindible en la solución de problemas a los que se verá enfrentado el estudiante en su futuro profesional (Silva y Sandoval, 2019).

Por lo tanto, el docente deberá actuar como agente facilitador de conocimientos y experiencias que ofrezcan el ambiente propicio, lo que genera procesos de enseñanza-aprendizaje mediados por herramientas TIC (Torres y Moreno, 2020). El factor de innovación en las prácticas y didácticas en el aula es altamente significativo para despertar interés en los estudiantes de la generación del siglo XXI. Se busca potencializar sus habilidades para afrontar diversas situaciones de manera reflexiva, crítica y constructiva. Por lo cual, se considera que dinamizar y potencializar el pensamiento lógico-matemático en los estudiantes de Ingeniería desarrolla sus competencias vinculadas a la adquisición de habilidades para proponer soluciones a diversas situaciones problemas. Asimismo, le permite fortalecer sus destrezas de razonamiento lógico y numérico, las cuales son capacidades esenciales que necesitará para desenvolverse en contextos dinámicos de su labor académica y su futuro ejercicio profesional. Esto puede influenciarse por medio de un software educativo que permita el fortalecimiento de estas capacidades matemáticas, al fomentar la exploración, interacción y construcción de conocimientos mediante una estrategia didáctica que busque motivar los aprendizajes de los estudiantes. 


\section{Software para el aprovechamiento de datos en las plataformas de aprendizaje haciendo uso de la minería de datos}

El objetivo del proyecto se orientó hacia el aprovechamiento de la información concebida por las interacciones de los estudiantes en las plataformas virtuales. Se planteó el desarrollo de una herramienta que permitiera gestionar la información generada por las interacciones de los estudiantes en las plataformas virtuales utilizando algoritmos para la minería de datos.

El diseño se fundamentó en la implementación de la metodología Cross Industry Standard Process for Data Mining (CRISP-DM). Esta se basa en la ejecución de seis fases, en las cuales se debe tener en cuenta: la identificación de las necesidades del cliente, ubicándose en la comprensión del negocio; luego debe realizarse un estudio y análisis de los datos. Estos constituyen las fases dos y tres del modelo. Una vez identificados los requerimientos se deben proponer los algoritmos más adecuados; esta fase se denomina modelamiento. Dicha fase se ratifica con la fase de evaluación, que permite constatar la aplicación de los modelos. Por último, está la fase de despliegue, en la cual se formatean los datos que se le presentarán al usuario final (IBM Knowledge Center, 2020).

Para el desarrollo del prototipo se realizó la implementación de diferentes algoritmos, los cuales se implementaron por medio de la herramienta WEKA. Estos fueron desarrollados en lenguaje Java con licencia (GLP), de libre uso, creada por la Universidad Waikato de Nueva Zelanda. Para determinar la operatividad del desarrollo, se realizó el entrenamiento de los archivos CSV generados en la plataforma Moodle, correspondiente a algunas interacciones de los estudiantes en la plataforma (WEKA, 2020).

Por otra parte, en el Semillero Green TIC, los integrantes han trabajado en diferentes actividades durante el periodo 2020-1 y 2020-2, dando respuesta al proyecto Diseño de una herramienta interactiva para la identificación de los estilos de aprendizaje de los estudiantes para el fortalecimiento y desarrollo de la educación a distancia, el cual tiene como finalidad el diseño de una aplicación web interactiva adaptable en el aula virtual del espacio académico de algoritmos de programación, que permite reconocer el estilo de aprendizaje de cada alumno mediante el uso del test de Felder y Silverman (1988). De lo anterior, desde el semillero cada auxiliar de investigación le apunta al desarrollo de algunas de las siguientes actividades, que dan respuesta al objetivo de la investigación: 1) caracterización de los estilos de aprendizaje; 2) diseño de la herramienta interactiva; y 3) Objeto Virtual de Aprendizaje (OVA).

\section{Caracterización de los estilos de aprendizaje de los estudiantes teniendo en cuenta el test de Felder y Silverman (1988)}

El objetivo del proyecto se orientó hacia la identificación de los enfoques dados desde la Teoría de los Estilos de Aprendizaje que permitan evidenciar las diferencias de adopción de conocimientos por parte de los miembros de la comunidad objeto de estudio, para mejorar los procesos formativos.

A pesar de que la terminología usada para definir los estilos de aprendizaje han variado de acuerdo con el autor referenciado, el concepto más acorde lo podemos retomar del trabajo publicado en la Revista Colombiana de Educación. Allí, Pantoja, Duque y Correa (2013) hacen una traducción de Keefe, James W., quien en su obra Learning Style Theory and Practice (1987) manifestó que los estilos de aprendizaje hacen referencia a los atributos cognitivos, afectivos y fisiológicos que sirven como indicador de la manera cómo las personas reconocen, percatan e interaccionan en su entorno de aprendizaje.

De lo anterior, los desafíos educativos en el mundo son cada día más elevados. Si bien, la educación ya resulta ser un proceso complejo al enfrentar a las personas a exigencias cognitivas durante las diferentes etapas de la vida, a lo largo de la época universitaria podría resultar más cómodo instaurar herramientas que promuevan el desarrollo integral de los estudiantes, mediante técnicas que potencialicen los conocimientos y permitan enfrentar los retos profesionales fácilmente.

Para ello, en búsqueda de fortalecer el desarrollo integral de los alumnos que pertenecen a las estructuras de programación en la modalidad a distancia de la Universidad Santo Tomás, se ha elegido el Modelo Felder y Silverman (1988) como metodología para identificar los estilos de aprendizaje que responden a las diferentes formas de potencializar la formación, así como: visualverbal (educación a través de diagramas, cuadros o sonidos), sensitivo (información recibida por sensaciones y de manera intuitiva), secuencial-global (educación de manera progresiva que necesita entendimiento integral de las cosas) y activo-reflexivo (información recibida mediante diálogos o discusiones). Una vez aplicado el modelo, sin duda, podrá evaluarse no solo el avance de los estudiantes en sus nuevos conocimientos adquiridos, sino que garantizará que los futuros graduados alcancen de manera segura un potencial individual en sus habilidades profesionales.

Para la investigación que nos atañe, nos ocuparemos de dar solución a un problema concreto. Para caracterizar la población que participará del estudio acudiremos a los planteamientos formulados por el profesor de ingeniería Richard Mark Felder y la doctora en psicóloga Linda Kreger Silverman en el año 1988 (Learning and Teaching Styles in Engineering Education).

Este índice de estilos de aprendizaje es un cuestionario de 44 preguntas, de las cuales solo hay dos opciones de respuesta y únicamente se puede elegir una respuesta para cada pregunta. Así mismo, para obtener un resultado fiel a la realidad se deben responder todas las preguntas a conciencia, ya que cuando se presente una situación en la cual ambas respuestas a una pregunta tiendan a aplicarse a la persona que resuelve el cuestionario, se debe considerar la que tenga mayor relevancia. El mismo Richard Felder realizó una actualización al modelo propuesto en 1988 (Felder R. M., 1993), que permite clasificar la manera en que se recibe y se procesa la información a través de la formulación de ciertas preguntas definidas, que con su respuesta catalogaban las siguientes dimensiones.

\section{Percepción sensitiva e intuitiva}

Los estudiantes que pertenecen a esta categoría divisan la información desde dos entornos: 1) el sensitivo, donde se clasifican aquellos que generan un gusto por aprender de los hechos, resolver problemas sin complicaciones con métodos concretos, memorizar los hechos, realizar prácticas y ser cuidadosos con los procedimientos, de igual manera generan una falta de interés por los cursos que no tienen relación con el mundo real; y 2) los intuitivos, que gustan de descubrir posibilidades y relaciones, innovar, aprender conceptos nuevos, trabajar con mayor rapidez, y pierden interés por los cursos que requieren de mucha memorización y cálculos frecuentes (Felder y Soloman, s.f., p. 2). Podemos entender entonces que los estudiantes sensitivos perciben la información a través de la vista, el oído o las sensaciones físicas, mientras que los intuitivos a través de recuerdos, ideas o percepciones. 


\section{Percepción visual y verbal}

Los estudiantes que pertenecen a esta categoría vislumbran la información más efectivamente desde dos medios: 1) el visual, donde logran una mayor absorción del conocimiento gráficamente, llevando a los instructores a no solo transmitir la información por recuentos literarios o la transcripción de texto, sino a reflejar dicha información en videos o medios audiovisuales interactivos; y 2) el verbal, que involucra no solo una transmisión por parte del instructor, sino la comprensión por parte del estudiante, quien debe discutir en grupo el material de estudio y trasladar los textos a sus propias palabras (Felder y Soloman, s. f., pp. 2-3). Podemos entender entonces que los estudiantes visuales perciben la información mediante imágenes, diagramas, gráficos o demostraciones; mientras que los verbales lo hacen mediante sonidos, expresión oral y escrita, o fórmulas.

\section{Percepción activa y reflexiva}

Los estudiantes que pertenecen a esta categoría procesan la información más efectivamente desde dos entornos: 1) el activo, que es cuando el estudiante desarrolla una tendencia a tomar la información y trabajar sobre ella, discutiéndola o retransmitiéndola a otras personas, probando los conocimientos adquiridos en el momento de trabajar en grupo; y 2) el reflexivo, en el que los estudiantes tienden a pensar sobre la información recibida y analizarla, esto en un ambiente de trabajo solitario que les permita recapacitar sobre lo aprendido (Felder y Soloman, s. f., p. 1). Podemos entender entonces que los estudiantes activos perciben la información a través del compromiso y en actividad física, no aprenden de conferencias, solo de la experiencia, el trabajo en grupo o las actividades que involucren hacer algo con la información recibida, discutirla, explicarla, probarla; mientras que para los reflexivos implica manipular la información realizando un análisis introspectivo.

\section{Percepción secuencial y global}

Los estudiantes que pertenecen a esta categoría progresan hacia el entendimiento desde los entornos: 1) secuencial, que lleva a los estudiantes a encontrar soluciones mediante pasos lógicos, lo que en ocasiones los lleva a buscar formas de tomar la información recibida y relacionarla con cosas conocidas para no perder el orden de los temas, sobre todo cuando el instructor tiene una metodología distinta a la practicada por ellos; y 2) los estudiantes globales que, en contraste, absorben la información de manera aleatoria y tienen la capacidad de relacionarla al final de la lección. Estos últimos además poseen la habilidad de hallar soluciones rápidas que, aunque poco fundamentadas, muestran un panorama general de la resolución. Por razones como esta es recomendable para estos estudiantes buscar contextualizarse de los temas a abarcar para tener una visión rápida del camino que se va a tomar en las lecciones, para luego encontrar las conexiones entre temas (Felder y Soloman, s. f., pp. 3-4). Podemos entender entonces que los estudiantes secuenciales avanzan en una progresión lógica de pasos incrementales pequeños, lineales, desde lo básico a lo más avanzado, mientras que para los globales involucra trabajar con material que les proporciona una comprensión parcial o superficial, en largos saltos, de manera intuitiva, holística.

\section{Software de entorno para la ejecución de estilo de aprendizaje}

El objetivo del proyecto es el diseño de una herramienta interactiva para el espacio académico de algoritmos de programación, la cual permita al estudiante reconocer su estilo de aprendizaje por medio del test de Felder y Silverman (1988), para que de esta forma puedan adquirir conocimientos de manera adecua.

De lo anterior, el diseño de la herramienta se estructurara desde una aplicación web, la cual fue denominada Code Learn y realizada en Java, Spring, Angular, MySQL y Okta como proveedor para el manejo de las credenciales. Esta aplicación le permite a los usuarios, docentes y estudiantes registrarse y acceder a ella a través de un login y contraseña segura. Después, los alumnos pueden responder el test de estilos de aprendizaje de Felder y Silverman (1988) (figura 3). Al finalizar, conocen el modo con el cual se identifican y adquieren conocimiento con mayor facilidad. Los docentes tienen módulos creados para que en el futuro puedan gestionar los estilos de aprendizaje y conocer los resultados del test de los estudiantes.

A continuación, se presenta la lista de componentes de la aplicación Code Learn:

- ' admin-code-learn': para el módulo de Administración de Estilos de Aprendizaje, el cual solo se muestra para los docentes. Es allí donde pueden gestionar todos los estilos de aprendizaje (añadir, editar).

- 'code-learn': para el módulo de Estilos de Aprendizaje, disponible en la aplicación para estudiantes y docentes. En este se pueden ingresar los contenidos de acuerdo con el estilo de aprendizaje que haya obtenido el estudiante.

- 'cuestionario': componente para insertar la lógica y el Front-End del cuestionario. Este módulo está disponible para los estudiantes y docentes.

- 'login': componente donde se encuentra la lógica de acceso a la aplicación a través de la conexión con Okta.

- 'reporte-estudiante': para el módulo de Reporte de Estudiante, en el cual debería estar visible para los docentes con el fin de acceder al listado de estudiante con el respectivo estilo de aprendizaje.

- $\quad$ user-profile': componente para que el usuario pueda visualizar su información básica en la aplicación. Este componente es para el módulo Perfil de Usuario dentro de la aplicación, el cual está disponible para usuario tipo estudiante y docente. 
Figura 3. Entorno de ejecución de estilo de aprendizaje

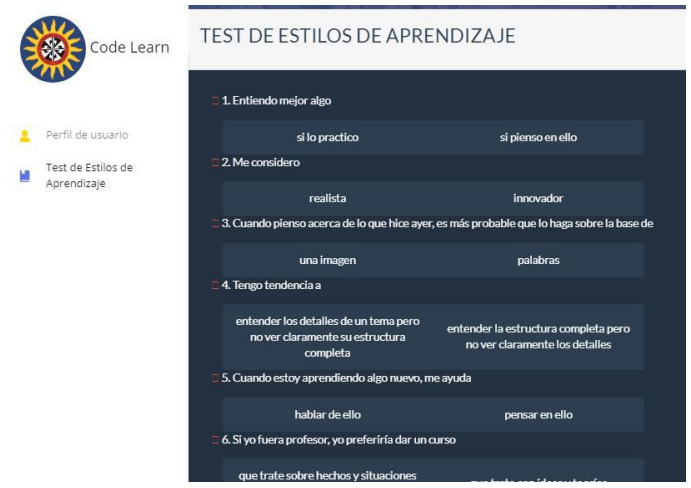

Fuente: CODE (2020).

Esta herramienta ha sido el fruto del Seminario Green TIC durante los últimos meses y es un gusto poner en práctica los conocimientos adquiridos durante los últimos años para entregarle a la Universidad Santo Tomás un producto de estudiantes para estudiantes. Esta ha sido una gran iniciativa y será realmente beneficioso que en el futuro cercano más estudiantes continúen trabajando en ella y pueda ser implementada en otras asignaturas y programas académicos.

\section{Diseño de objetos virtuales de aprendizaje (OVA) para el estilo de aprendizaje visual- verbal}

El objetivo del proyecto se encaminó al diseño de la interfaz interactiva comprendida por objetos virtuales de aprendizaje, con las cuales los estudiantes podrán adquirir conocimiento. De lo anterior, las interfaces u objetos virtuales de aprendizaje (OVA) se diseñaron de acuerdo con la metodología de los diversos estilos de aprendizaje basados en el sistema de Felder y Silverman (1988), enfocando al estilo de aprendizaje visual-verbal. Para este, en cada módulo de aprendizaje desarrollado se encuentran diversos recursos y herramientas que permiten la correcta apreciación y comprensión de la información por parte del estudiante que está adquiriendo conocimientos mediante esta herramienta (figura 4).
El OVA de estilo de aprendizaje visual-verbal se compone de: 1) inicio, donde se podrá visualizar la presentación de la herramienta, las competencias y resultados de aprendizaje a desarrollar y la metodología; 2) síntesis del contenido, el cual presenta la estructura en forma de mapa mental del objeto virtual de aprendizaje; 3 ) situación problémica, donde el estudiante podrá conocer la problemática a trabajar en las diferentes unidades referentes a un contexto del día a día que vive la familia Gutiérrez Rodríguez; y 4) unidades temáticas, las cuales se componen de cinco contenidos: introducción, condicional básico, condicional si no, condicional con doble condición al mismo nivel y condicional con doble condición o múltiple condición en múltiples niveles. El estudiante, de acuerdo con su método de estudio, puede aprender, practicar e ir fortaleciendo una y otra vez los conocimientos del condicional si y sus variantes. La herramienta también le permite afianzar los conocimientos adquiridos teóricamente mediante entornos gráficos de aprendizaje, ejemplos conceptuales, prácticos, interactivos y evaluativos. Esto constituye un recurso importante para apoyar la enseñanza centrada en el tipo de aprendizaje de cada uno de los estudiantes. El componente 5 del OVA corresponde al examen final, en el que se identifican dos momentos de evaluación: cuestionario y actividad de aprendizaje. En el primero se encuentran ocho preguntas de selección múltiple, que dan respuesta a las diferentes unidades vistas. En la actividad de aprendizaje se presentan dos problemáticas, a las cuales deben dar solución por medio de herramientas externas. 
Figura 4. OVA: estilo de aprendizaje Visual-Verbal
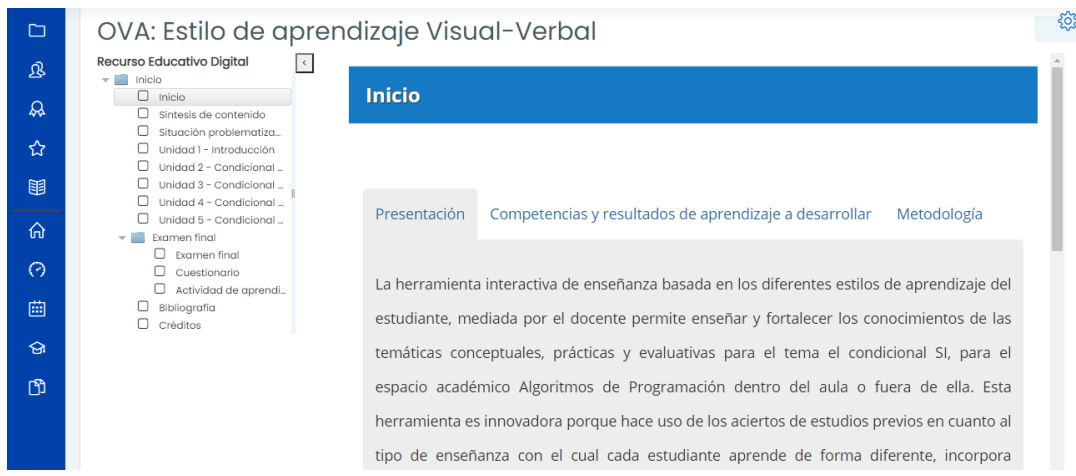

Fuente: USTA Distancia (2020).

Elementos clave como diagramas, audios, videos y documentación, le permiten al estudiante comprender con un alto grado de fluidez los temas incluidos en un plan de estudios impartidos por el docente, lo que permite que se aclaren dudas con rapidez, incluso en temas complejos que se aborden en el desarrollo de la materia. Por esto, la herramienta Code Learn permitirá evaluar el método de aprendizaje en el cual los estudiantes obtienen conocimiento con respecto al estilo de aprendizaje que más se acomode a su diario vivir (figura 5).

Figura 5. OVA: estilo de aprendizaje visual-verbal
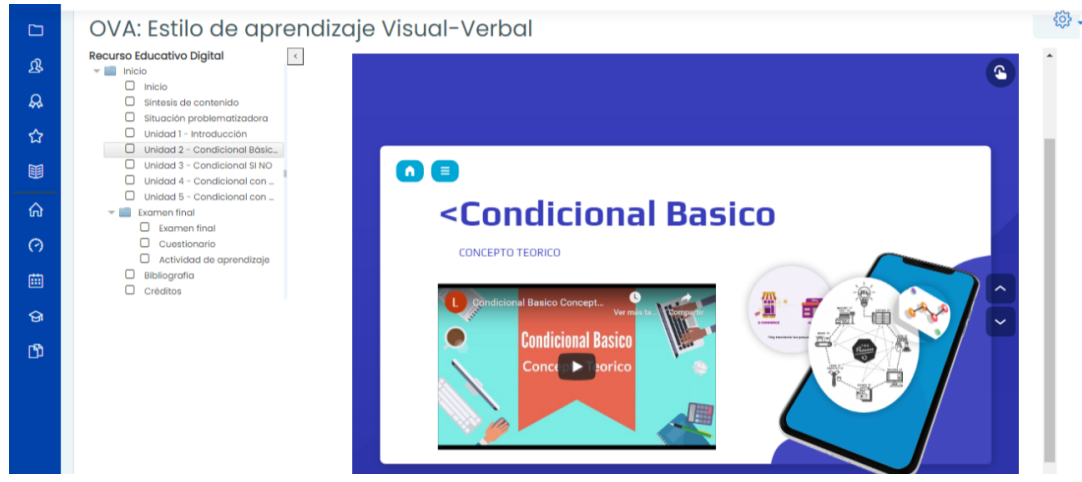

Fuente: USTA Distancia (2020).

\section{Conclusiones}

La investigación es vista como el norte de todo proceso de formación y de creación en el contexto del conocimiento. A nivel educativo, conocer se convierte en el primer paso para hacer investigación y, a través de esta, explicar los acontecimientos que ocurren en los contextos sociales, políticos, económicos y culturales en que se presentan, que están interfiriendo de manera directa con los cambios educativos implementados en las instituciones colombianas. De esta forma, la investigación formativa permite llegar a universalizar el conocimiento; también lleva a los investigadores a buscar metodologías que puedan mostrar el camino en el proceso de enseñanza-aprendizaje. Esto nos acerca a la investigación formativa y nos saca de los esquemas tradicionales epistemológicos para enfrentar hechos reales de la comunidad en general.
Esta nueva noción demanda congruencia con los preceptos de la Ley General de Educación (Ley 115 de 1994), que constituye, para el sistema de formación profesional en nuestro país, centralizar los procesos pedagógicos en el perfeccionamiento sistémico de las capacidades y aptitudes de las personas. Esto emprende un proceso de vida universitaria que permita alcanzar experiencias que exigen responsabilidades intelectuales y ético-políticas consigo mismo, con sus colectivos y con su entorno sociocultural. Esto permite transmutar al estudiante en un autodidacta que requiere continuamente innovar en los procesos de investigación para enriquecer sus saberes previos.

Las instituciones educativas se orientan en la formación integral de los estudiantes; de esta manera, los semilleros de investigación se orientan hacia el fortalecimiento de habilidades investigativas. Con esto se pretende contribuir al avance de la ciencia y la tecnología, y su integración en contextos educativos, donde es posible evidenciar que los trabajos adelantados en los semilleros de 
investigación permiten el desarrollo de habilidades investigativas, lo que incentiva la formulación de propuestas innovadoras fundamentadas en las disciplinas de la informática. En este sentido, se propende por la construcción colectiva de capacidades en investigación formativa mediante la estrategia de semilleros. Cada uno de los resultados de los trabajos presentados por los estudiantes ha contribuido a solucionar diferentes necesidades. De esta manera es posible determinar cómo la informática, por medio de sus diferentes disciplinas, puede aportar por el desarrollo de la sociedad.

\section{Referencias}

Code.org. (2020). Projects. https://studio.code.org/projects/public

Castiblanco, S. y López, L. (2020). Semilleros de investigación en educación virtual. Citas, 21-47. http://soda.ustadistancia.edu.co /enlinea/citas/edi5/files/assets/basic-html/page-27.html

Felder, R. M. (1993). Reaching the Second Tier: Learning and Teaching Styles in College Science Education. J. College Science Teaching. 23(5), 286-290. https://www.engr.ncsu.edu/wp-content/uploads/drive /1g7mzNhke6ErAkNXsQlyxBsmkaR-m8oe-/1993-Secondtier. pdf

Felder, R. M. y Silverman, L. K. (1988). Learning and Teaching Styles in Engineering Education. Engr. Education. 78 (7), 674-681. https://www.engr.ncsu.edu/wp-content/uploads/drive /1QP6kBI1iQmpQbTXL-08HS10PwJ5BYnZW/1988-LS-plusnote.pdf

Felder, R. M. y Soloman, B. A. (s. f.). Learning styles and strategies. https://www.engr.ncsu.edu/wp-content/uploads/driv e/1WPAfj3j5o5OuJMiHorJ-lv6fON1C8kCN/styles.pdf

Flórez, J., Aguilera, M. y Salcedo, O. (2019). Industria 4.0: tendencias de la literatura académica reciente. Espacios. 40, 27.

Hernández, R., Fernández, C. y Baptista, L. (2014). Metodologías de la Investigación. McGrawHill.

IBM Knowledge Center. (2020). Conceptos básicos de ayuda de CRISP. DM. https://www.ibm.com/support/knowledgecenter/e s/SS3RA7_sub/modeler_crispdm_ddita/clementine/crisp_help/ crisp_overview.html

Ministerio de Ciencia (Minciencias). (2020). Ecosistema Científico. https://minciencias.gov.co/investigadores/direccionde-fomento/estrategias

Ministerio de Ciencia (Minciencias). (2019). Misión de sabios. htt ps://minciencias.gov.co/mision_sabios

Pantoja, M. A., Duque, L. I. y Correa, J. S. (2013). Modelos de estilos de aprendizaje: una actualización para su revisión y análisis. Revista Colombiana de Educación. 64, 79-105. http:// www.scielo.org.co/pdf/rcde/n64/n64a04.pdf

Restrepo, B. (2004). Formación Investigativa $e$ investigación Formativa: Acepciones y Operacionalización de esta última. Universidad Distrital. http://planmaestroinv.udistrital.edu.co/documentos/P MICI-UD/InvestigacionFormativa/Formaci\%C3\%B3n\%20Inv estigativa\%20e\%20investigaci\%C3\%B3n\%20Formativa.pdf

Silva, A. y Sandoval, M. (2019). Organizadores gráficos: estrategia didáctica en ambientes virtuales mediada por la identificación de estilos de aprendizaje. Citas, 89-108. http://soda.ustadistanc ia.edu.co/enlinea/citas/edi5/files/assets/basic-html/page-5.html
Torres, C., y Moreno, G. (2020). Inclusión de las TIC en los procesos de enseñanza y aprendizaje universitario. Apertura, 48-65.

Universidad Santo Tomás. (2020). La formación de capacidades en CTeI: un compromiso con la excelencia académica. https://udcfd.usta.edu.co/images/III_encuentro_aca demico/retos/reto2-4.pdf

Villalba, J. C. y González, A. (2017). La importancia de los semilleros de investigación. Prolegómenos Derechos y Valores. 20(39), 9-10. https://doi.org/10.18359/prole.2719

USTA Distancia. (2020). OVA estilo de aprendizaje visual-verbal. https://www.ustadistancia.edu.co/

Weka. (2020). The workbench for machine learning. https://www .cs.waikato.ac.nz/ml/weka/

\section{Bibliografía consultada}

Clavijo, S. (2019). El semillero de investigación como herramienta didáctica para la formación de sujetos críticos de la historia local. Ciencia Nueva. Revista de Historia y Política, 3, 15. http s://doi.org/10.22517/25392662.20861

Observatorio del Tecnológico de Monterrey. (2019, septiembre 3). WhatsApp para la educación. https://observatorio.tec.mx/edubits-blog/whatsapp-para-la-educacion 